\title{
Coupled Vibration of Unshrouded Centrifugal Compressor Impellers. Part II: Computation of Vibration Behavior*
}

\author{
DIRK HAGELSTEIN ${ }^{\dagger}$, HEINRICH HASEMANN and MANFRED RAUTENBERG \\ Institute of Turbomachinery, University of Hannover, Appelstr. 9, D-30167 Hannover, Germany
}

(Received 12 May 1998; In final form 21 July 1998)

\begin{abstract}
The increased use of small gas turbines and turbochargers in different technical fields has led to the development of highly-loaded centrifugal compressors with extremely thin blades. Due to high rotational speed and the correspondingly high centrifugal loads, the shape of the impeller hub must also be optimized. This has led to a reduction of the thickness of the impeller disc in the outlet region. The thin parts of the impeller are very sensitive and may be damaged by the excitation of dangerous blade vibrations.

Experimental investigations of coupled vibration behavior are presented in Part $I$ of the paper. The results of these investigations were able to explain the dominance of coupling effects between the blades and the disc.

Part II of the paper presented here describes finite element (FE) code computations of the vibration behavior of the blades and the complete impeller for two different impeller types one with radial-ending blades and the other with backswept blades. The results are able to demonstrate the influence of coupling on dynamic behavior and the high importance of careful impeller design. The computational results were found to correspond very well with the results of the experimental investigations.
\end{abstract}

Keywords: Centrifugal compressor, Blade vibrations, Coupled vibrations, Finite element computation

\section{INTRODUCTION}

Owing to their large density concentrations, high efficiencies and compact design, highly-loaded centrifugal compressor impellers find wide application in an ever-increasing number of technical fields. Irrespective of whether these compressors are deployed as self-contained units or as modules in complex machines, a high degree of operational safety is expected of the impellers as outage times due to mechanical damage of the latter unavoidably lead to high costs. Operational practice has shown that damage to such impellers is very often linked to blade damage as a direct result of vibration loading.

* This paper was originally presented at ISROMAC-7.

${ }^{\dagger}$ Corresponding author. Tel.: (0511) 762 2737. Fax: (0511) 7622768. 
Owing to the required high peripheral speed, these impellers must be designed with very thin blades in order to ensure that the loading due to centrifugal forces lies within permissible limits. This results in very flexible blades which are highly prone to vibration excitations.

The development of high-performance computers and corresponding software has made it possible to compute the natural vibration behavior of impellers with the aid of the finite element (FE) method. Previous investigations by Haupt (1984) and Jin (1990) have indicated, however, that a consideration of individual blades only in the computations is insufficient to satisfactorily reproduce the actual vibration behavior. Coupled action between the extremely thin outer portion of the impeller disc and the individual blades greatly influences the vibration behavior of the overall system as well as its characteristics. Coupling between the blades and the relatively thin and hence flexible part of the impeller disc in the outlet region results in mutual interaction between the vibration behavior of the blades and the disc, which can only be modeled by considering the overall system. The fact that FE models of complete impellers lead to problems of storage capacity and computing time even in the case of large computers, it is often necessary to restrict the computations to reduced structures. The aim of the present investigation is to examine the coupled vibration behavior of centrifugal impellers.

\section{COUPLED VIBRATION}

Before examining the impeller models, a brief account will first be given of several theoretical aspects of the nature of the coupled vibration behavior in order to better understand the behavior of the structures to be dealt with at a later stage. A schematic representation of the mutual interaction between the blades and the disc in the outlet region of the impeller is shown in Fig. 1. In this diagram the impeller disc runs in the $x$-direction while the outlet edges of the blades run in the $y$-direction. The disc exhibits sinusoidal vibrations with antinodes located in each case between two blades and nodes at each blade root. Corresponding to the motion of the impeller disc, adjacent blades thus vibrate in the opposite sense. This clearly demonstrates the mutual interaction between the blades and the disc. By way of example, Fig. 2 shows a section of a computed mode shape with significant participation of the impeller ring. Good agreement with the sketch of Fig. 1 is clearly evident.

The problem of computing coupled vibration systems comprised of $z$ individual components is that for $n$ natural frequencies of the individual structure $z * n$ natural frequencies of the overall system must be computed as each natural frequency

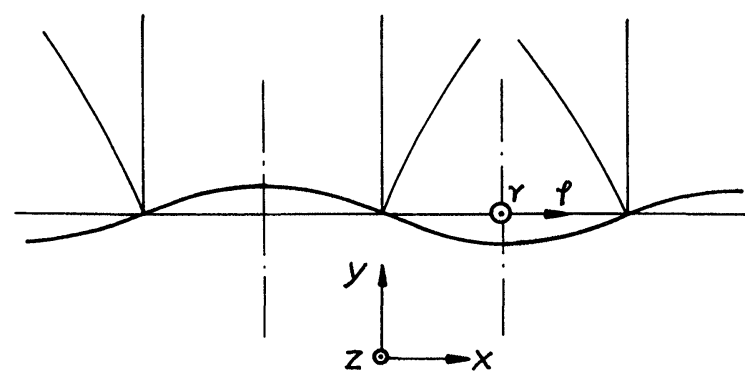

FIGURE 1 Schematic coupling mechanism between the blades and the disc in the outlet region of the impeller.

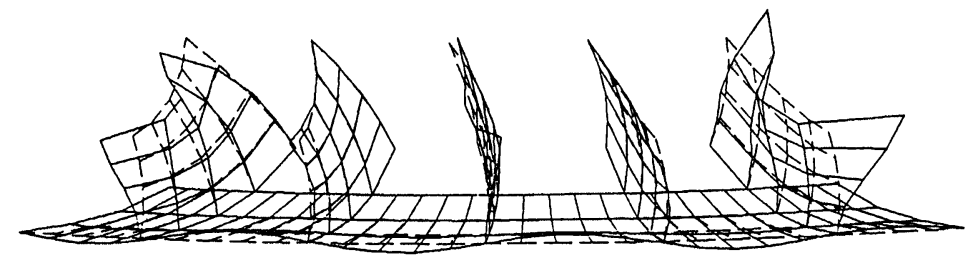

FIGURE 2 Computed coupling mechanism between the blades and the impeller disc. 
occurs $z$ times in the coupled vibration system. It should be noted, however, that the same numerical value is not computed exactly $z$ times due to the fact that coupling effects lead to the formation of frequency bands in which $z$ natural frequencies are spread. This will be illustrated by considering a coupled vibration system with two degrees of freedom.

The diagram presented in Fig. 3 shows a springmass system comprised of two coupled single oscillators. The following fundamental dynamic equations may be formulated for the disconnected masses in the right-hand part of the figure:

System 1:

$$
\ddot{x}_{1}+\omega_{1}^{2} x_{1}-\frac{c_{1,2}}{M_{1}} x_{2}=0 \text { with } \omega_{1}^{2}=\frac{c_{1}+c_{1,2}}{M_{1}} .
$$

System 2:

$$
\ddot{x}_{2}+\omega_{2}^{2} x_{2}-\frac{c_{1,2}}{M_{2}} x_{1}=0 \text { with } \omega_{2}^{2}=\frac{c_{2}+c_{1,2}}{M_{2}}
$$

$\omega_{1}$ and $\omega_{2}$ represent the natural frequencies of the uncoupled Systems 1 and 2, respectively. The above equations represent a system of two coupled, linear, homogeneous differential equations of the second order with constant coefficients. The general solutions of these equations are given by:

$$
x_{1}=A_{1} \cos \left(\omega_{\mathrm{k}} t\right) \text { and } x_{2}=A_{2} \cos \left(\omega_{\mathrm{k}} t\right),
$$

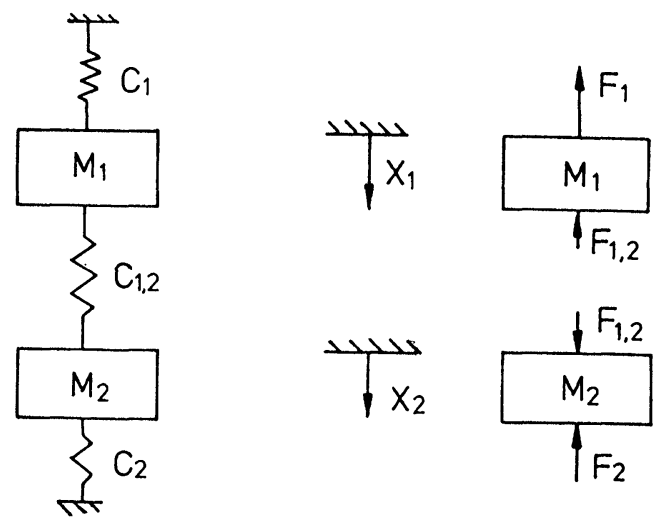

FIGURE 3 Spring-mass system comprised of two coupled oscillators. where $A_{1}$ and $A_{2}$ represent the amplitudes and $\omega_{\mathrm{k}}$ the coupled angular frequencies of the coupled vibrations. Substituting the general solutions into the differential equations yields:

$$
\begin{aligned}
& -A_{1} \omega_{\mathrm{k}}^{2}+\omega_{1}^{2} A_{1}-\frac{c_{1,2}}{M_{1}} A_{2}=0 \quad \text { and } \\
& -A_{2} \omega_{\mathrm{k}}^{2}+\omega_{2}^{2} A_{2}-\frac{c_{1,2}}{M_{2}} A_{1}=0 .
\end{aligned}
$$

This homogenous system of equations for $A_{1}$ and $A_{2}$ only has a non-trivial solution provided that:

$\operatorname{det} \underline{A}=0 \Rightarrow\left[\omega_{1}^{2}-\omega_{\mathrm{k}}^{2}\right] \times\left[\omega_{2}^{2}-\omega_{\mathrm{k}}^{2}\right]-\frac{c_{1,2}^{2}}{M_{1} M_{2}}=0$.

The solution of this biquadratic equation is given by:

$$
\begin{aligned}
\omega_{\mathrm{k}}^{2}= & \frac{\omega_{1}^{2}+\omega_{2}^{2}}{2} \\
& \pm \sqrt{\left[\frac{\omega_{1}^{2}+\omega_{2}^{2}}{2}\right]^{2}-\omega_{1}^{2} \omega_{2}^{2}+\frac{c_{1,2}^{2}}{M_{1} M_{2}}} .
\end{aligned}
$$

Taking all masses and spring constants to be equal in analogy to the impellers considered here with identical single blade structures:

$$
M_{1}=M_{2}=M \quad \text { and } \quad c_{1}=c_{2}=c_{1,2}=c
$$

results in the following coupled natural frequencies:

$$
\omega_{\mathrm{k} 1}=\sqrt{\omega^{2}-\frac{c}{M}} \text { and } \omega_{\mathrm{k} 2}=\sqrt{\omega^{2}+\frac{c}{M}},
$$

whereby the following holds under the above conditions:

$$
\omega^{2}=\frac{2 c}{M}
$$

The coupled natural frequencies may thus be written as:

$$
\omega_{\mathrm{k} 1}=\sqrt{\frac{c}{M}} \text { and } \quad \omega_{\mathrm{k} 2}=\sqrt{3 \frac{c}{M}} .
$$


It thus follows that:

$$
\omega_{\mathrm{k} 1}<\omega<\omega_{\mathrm{k} 2}
$$

This result clearly indicates that the coupled natural frequencies form a frequency band which is wider than the natural frequency spectrum of the individual oscillators. Moreover, it is seen that 2 individual components give rise to $2 * n$ coupled natural frequencies. As also illustrated by the example computed above, completely identical individual structures with identical natural frequencies give rise to frequency bands in the coupled state. If the natural frequencies of the individual structures are slightly different, e.g. due to the manufacturing process, this leads to further spreading of the coupled frequency bands.

The results of the coupled vibration behavior presented here for the example of a 2-mass oscillator may be directly applied to larger systems. For the case of the impellers investigated in the present study, the masses represent greatly simplified substitute systems for the blades whereas the disc is modeled by the spring elements. For an impeller with 20 identical blades, 20 coupled natural frequencies may therefore be expected in each frequency band.

\section{FE MODELING}

In order to apply the FE method for computing the impeller vibration behavior it is first necessary to generate a computational mesh. This requires a mathematical description of the structures in question so as to achieve the best possible representation of the actual impeller in the FE model.

As both impellers are comprised of normal and splitter blades, it was first necessary to generate FE meshes for both types of blade. It was also necessary to generate a mesh for the annular outer portion of the hub for both types of impeller. The individual structures were finally combined to form the complete impeller models.

As the blade types of both impellers as well as the impeller discs in the outlet region relevant to the computations are very thin, shell elements were adopted for the FE computations.

As the blades are firmly attached to the hub along their inner profiles, all degrees of freedom were suppressed along the common line of attachment. This is equivalent to a rigid fixture of the blade root and represents a close approximation to actual conditions, as the hub may be treated as a massive, rigid body. Strictly speaking, however, this only applies from the inlet region to about two thirds along the blade length as the massive hub reduces to a thin flexible disc in the outlet region of the impeller. The blade fixture is much more flexible in this region and cannot be realistically modeled by suppressing all degrees of freedom. In order to achieve a better approximation to the actual fixture conditions, only a part of the impeller disc was modeled in the outlet region of the blades (see Fig. 4). The model is thus comprised of the blades attached to a thin annular portion of the impeller disc in the outlet region.

\section{The $90^{\circ}$-Impeller}

The $90^{\circ}$-impeller consists of 28 radial-ending blades with every second blade cut back at the inlet. As the

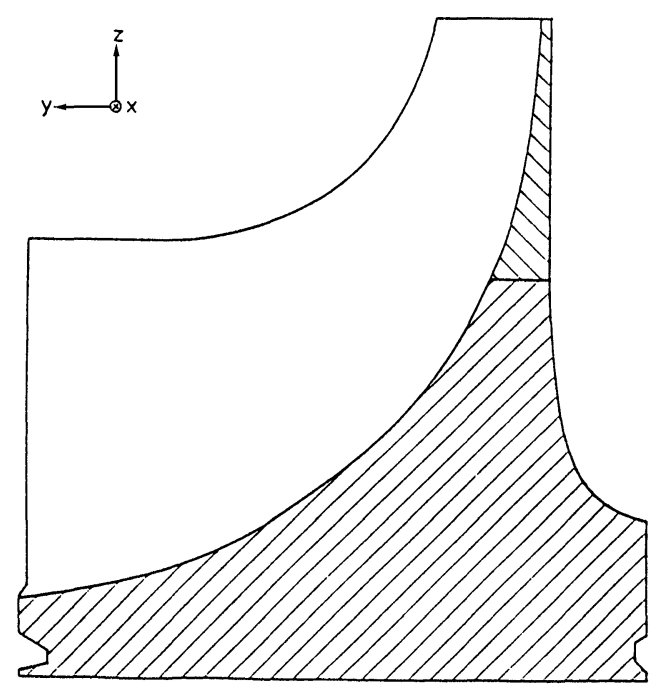

FIGURE 4 Hub body with meridional section of a blade indicating the rigid impeller body and the flexible impeller back wall. 
impeller was manufactured by the profile milling method, mathematical data capable of being directly evaluated for generating the mesh were not available. It was therefore necessary to develop the profiles of the normal and splitter blades based on drawings and construction plans and match these to the requirements of the FE program. Subsequent reference measurements on the manufactured impeller served for checking and correcting the generated meshes.

The leading edge of the splitter blade is cut back by $39 \mathrm{~mm}$ compared with the leading edge of the normal blade, both of which lie exactly on radial rays. The root of the normal blade as well as the splitter blade was attached to a segment of the impeller disc in the outlet region.

Besides the normal and splitter blades, the flexible impeller outlet region also plays a very important role in the coupled vibration behavior of the impeller. The impeller disc represents the coupling medium and has a significant influence on the vibration behavior of the impeller. This means, of course, that a knowledge of the natural vibration behavior of the impeller disc is absolutely essential.

In configurating the impeller disc of the $90^{\circ}$ impeller, the nodal coordinates of the hub profile of the blade along the corresponding attachment length were adopted and a curved, closed annular disc was generated by applying an angular rotation about the impeller axis. Adoption of the nodal coordinates guaranteed the unproblematic interconnection of the blades with the impeller disc for generating the complete model of the impeller. Shell elements were also used for modeling the impeller disc structure as the outer part of the disc under consideration could also be treated as a thin shell.

In order to generate a model capable of reproducing coupled vibrations, the previously generated blade models for the normal and splitter blades, each of which are attached to an annular section of the impeller disc at the outlet, were linked together in alternation. This involved the transformation of the existing cylindrical nodal coordinates so as to obtain the new peripheral position on the impeller.

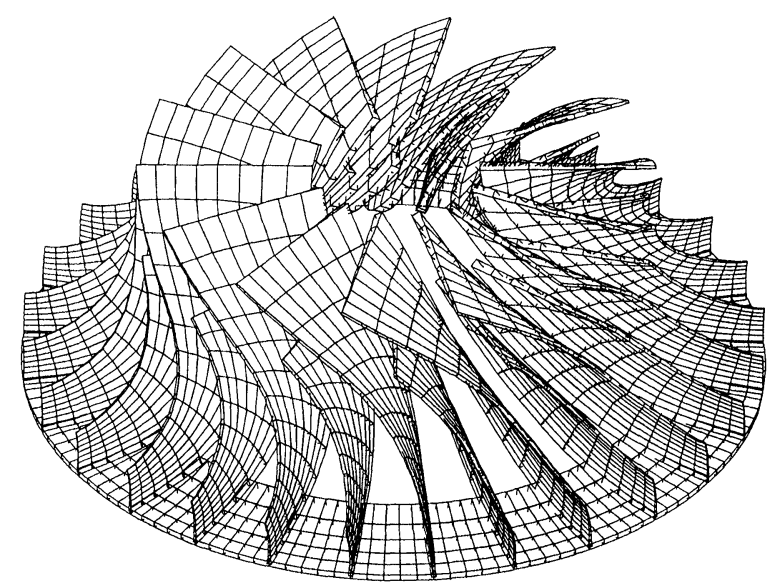

FIGURE 5 Complete FE model of the 28-bladed $90^{\circ}$-impeller.

This procedure permits the generation of impeller sub-models as well as complete impeller models.

Figure 5 shows the model of the 28-bladed $90^{\circ}$-impeller generated in this manner. In this representation the thickness information assigned to each node is also plotted to create a 3-dimensional impression.

\section{The $60^{\circ}$-Impeller}

In contrast to the impeller with radial-ending blades, the $60^{\circ}$-impeller consists of only 20 backswept blades. Every second blade is again cut back at the inlet in order to minimize blockage. In the case of this impeller the reduction to 20 blades as well as cutting back is also necessary on account of the manufacturing method used. The $60^{\circ}$-impeller was manufactured by flank milling on a numerically-controlled 5-axis CNC machine. As suggested by the name, the milling tool in this method cuts along its entire flank. This method offers the enormous advantage of clean, exact and quick manufacturing, enables the realization of highgrade surfaces without the need for post-machining and is ideally suited to automation technology. Owing to the high cutting forces associated with flank milling and the correspondingly high mechanical loading of the milling head, certain restrictions 
are imposed on the minimum permissible thickness of the cutting tools. This dictates the smallest possible free admission area between the blades at the inlet and hence automatically determines the maximum number of blades possible.

The generation of the normal blade profiles of the $60^{\circ}$-impeller was carried out on the basis of ruled surfaces. The exact mathematical description of the blade profiles by means of ruled lines after Rautenberg et al. (1989a,b) also enables the FE meshes to be generated in a very flexible manner. Analagous to the procedure already mentioned for the $90^{\circ}$-impeller, a structure was generated in which the blades in the hub region of the outlet rest on a portion of the impeller disc. As the skeleton lines of the normal and splitter blades are identical and only differ in terms of the thickness distribution, the mesh for the splitter blade could be generated in exactly the same way as for the normal blade. The fineness of the discretization was suitably matched to the subsequent coupling of the individual structures. All degrees of freedom were suppressed at the nodes along the hub profile. In order to simulate the flexible fixture on the impeller disc, an annular portion of the disc in the outlet region was generated in a similar manner to that already described for the $90^{\circ}$-impeller.

In order to generate the complete model of the $60^{\circ}$-impeller the blade roots of the existing normal and splitter blade models were alternately attached to the impeller disc. This required a corresponding conversion of the nodal cylindrical coordinates with subsequent matching of the node and element numbers as well as the thickness allocations. Moreover, the nodal degrees of freedom were appropriately matched to the corresponding boundary conditions.

The resulting model of the $60^{\circ}$-impeller with 20 blades is shown in Fig. 6. In this representation the thickness information assigned to each node is plotted to create a 3-dimensional impression. As in the case of the $90^{\circ}$-impeller model, the inner edge of the annular impeller disc as well as the blade nodes along the hub profile were rigidly fixed by suppressing all degrees of freedom.

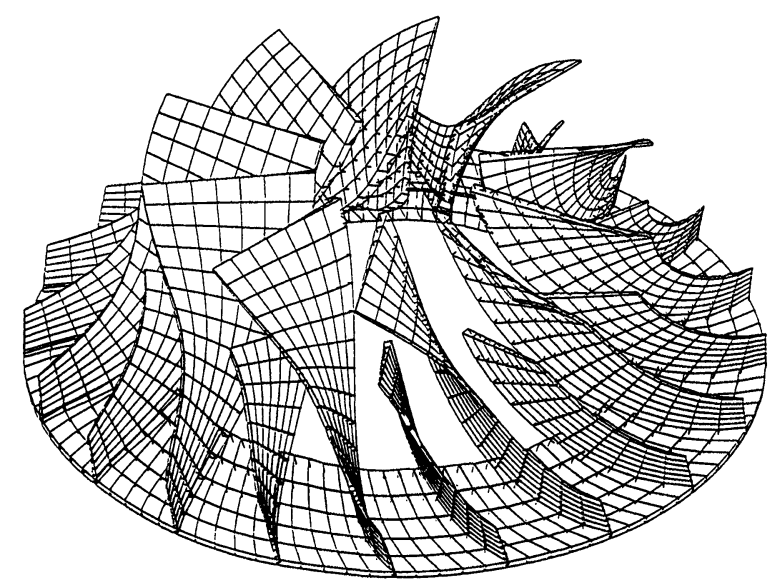

FIGURE 6 Complete FE model of the 20-bladed $60^{\circ}$-impeller.

\section{RESULTS OF THE CALCULATIONS}

Figure 4 shows the hub body of the impeller through a meridional section of a blade. The flexible annular portion of the hub is indicated by a different type of shading. This clearly indicates the rapid increase in the thickness of the impeller disc with decreasing radius, which gradually merges with the massive hub body. As this portion of the impeller disc greatly influences coupled vibration behavior, the vibration behavior of this annular structure in isolation will first be investigated.

\section{The Disk}

Models of the bladeless outer portion of each impeller were obtained by generating annular discs rigidly fixed along their inner edges. Generation of the meshes was based on the number of blades in each case as well as the individual blade structures. The mode shapes of the $90^{\circ}$-disc including the corresponding node lines are shown in Fig. 7. The fundamental mode of vibration is analogous to an umbrella vibration, i.e. the entire outer portion of the disc vibrates in the same way as an opening and closing umbrella at right angles to the plane of the disc. 


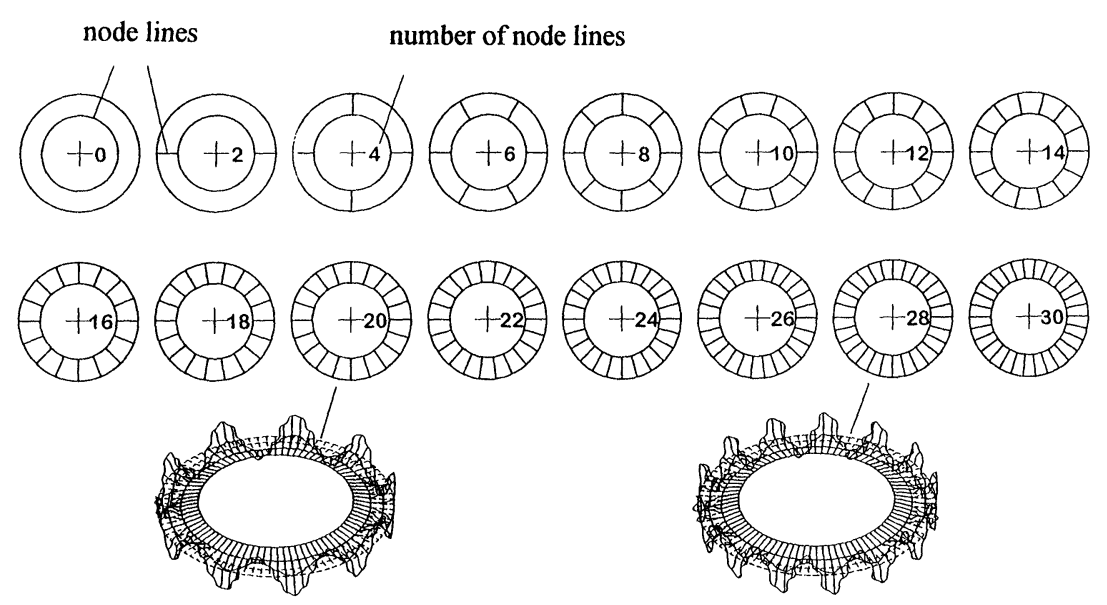

FIGURE 7 Mode shapes of the impeller ring rigidly fixed along its inner edge.

The risk of dangerous coupled vibrations always arises when the number of node lines corresponds to the number of blades. In this case the disc vibration is hardly suppressed by the blades as these essentially act as stiffeners along the node lines of the disc. This may ideally be the case for the $90^{\circ}$-impeller as the blade paths as well as the node lines run purely radially. Due to the impeller outlet angle of $60^{\circ}$ the curved blade path of the $60^{\circ}$-impeller, on the other hand, does not coincide exactly with the node lines. As already mentioned, however, amplified vibrations may also be transferred via the translatory degrees of freedom especially in the axial direction. A resonant vibrating impeller outlet region with a mode shape comprised of 20 or 28 node lines in turn excites the blades via coupling with the disc to produce large vibrations. If this excitation frequency is close to a blade natural frequency, there is a danger that the blade also exhibits resonance behavior with high material loading. In the case of the 28-bladed $90^{\circ}$-impeller the disc has exactly 28 node lines at a frequency of $5694 \mathrm{~Hz}$. According the FE computations of the individual structures the 8 th natural frequency at $5502 \mathrm{~Hz}$ and the 9th natural frequency at $5842 \mathrm{~Hz}$ lie close to this disc mode shape. Considering also the 20-bladed $60^{\circ}$-impeller, the disc mode shape with 20 node lines at $3782 \mathrm{~Hz}$ is also likely to pose a danger. According to the computational results for the normal blade structure, the $3 \mathrm{rd}$ natural frequency at $3624 \mathrm{~Hz}$ lies close to this disc natural frequency.

\section{Vibration Frequencies}

The experimental determination of coupled vibration behavior was carried out in the same manner as already described in Part I for the $90^{\circ}$-impeller. In this case also, the impeller at rest was first excited by means of a shaker. One strain gage was applied to a normal blade and a second to the impeller disc between two blades at the outlet. The results of strain gage measurements on the normal blade and at the outlet are shown under B in Figs. 8 and 9, respectively. These are compared with the corresponding computed results for the $60^{\circ}$ half-impeller with thickness variation under $\mathrm{A}$ in the figures.

As expected, the first natural frequency indicated in Fig. 8 was best detected by the strain gage and reproduced with the largest amplitude. The computations are found to agree well with the fundamental frequency as well as the 2nd natural frequency. The computations yield excessively high values in the case of the 3rd natural frequency whereas the mixed form as well as the 4th natural frequency are in close agreement with 


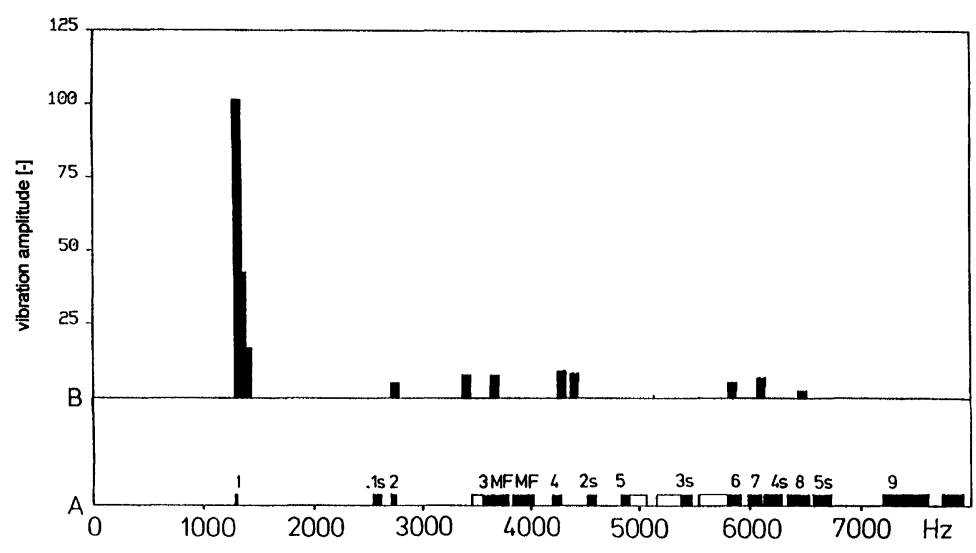

FIGURE 8 Coupled vibration frequencies of the 20 -bladed $60^{\circ}$-impeller. Comparison between the FE computational results (A) for the part model with 10 blades and measurements (B) for the impeller at rest with a strain gage on the normal blade.

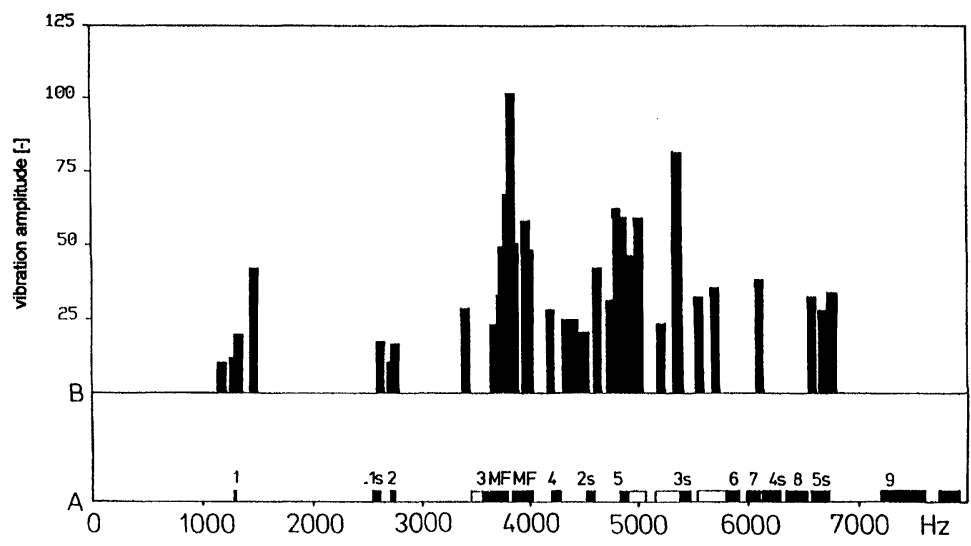

FIGURE 9 Coupled vibration frequencies of the 20-bladed $60^{\circ}$-impeller. Comparison between the $\mathrm{FE}$ computational results (A) for the part model with 10 blades and measurements (B) for the impeller at rest with a strain gage on the impeller disc.

measurements. The same applies to the 6th, 7 th and 8th natural frequencies, which are also satisfactorily reproduced by the computations. The 5 th mode shape was not registered by measurements.

In the case of the strain gage measurements on the impeller disc shown in Fig. 9 it is seen that the middle and higher frequencies are better detected. In this case the natural frequencies of the splitter blades are also registered by the measurements. Beginning with the mixed forms (MF) at $3600 \mathrm{~Hz}$ up to about $5000 \mathrm{~Hz}$ it is seen that the impeller disc also participates to a large extent in the overall vibration behavior, as indicated by pronounced coupled vibrations.
Apart from the measurements with the impeller at rest, blade vibration measurements were also carried out on the running impeller. As already described in Part I for the $90^{\circ}$-impeller, the signals from the semiconductor strain gages were transmitted by means of a telemetry transmitter, recorded and analyzed. The results of measurements as well as the computed results for the halfimpeller model with thickness variation were again plotted in a Campbell diagram.

Figure 10 shows the measurements obtained from a strain gage applied to the normal blade in the region of maximum stress corresponding to the 1st mode shape. The shaded areas represent the 


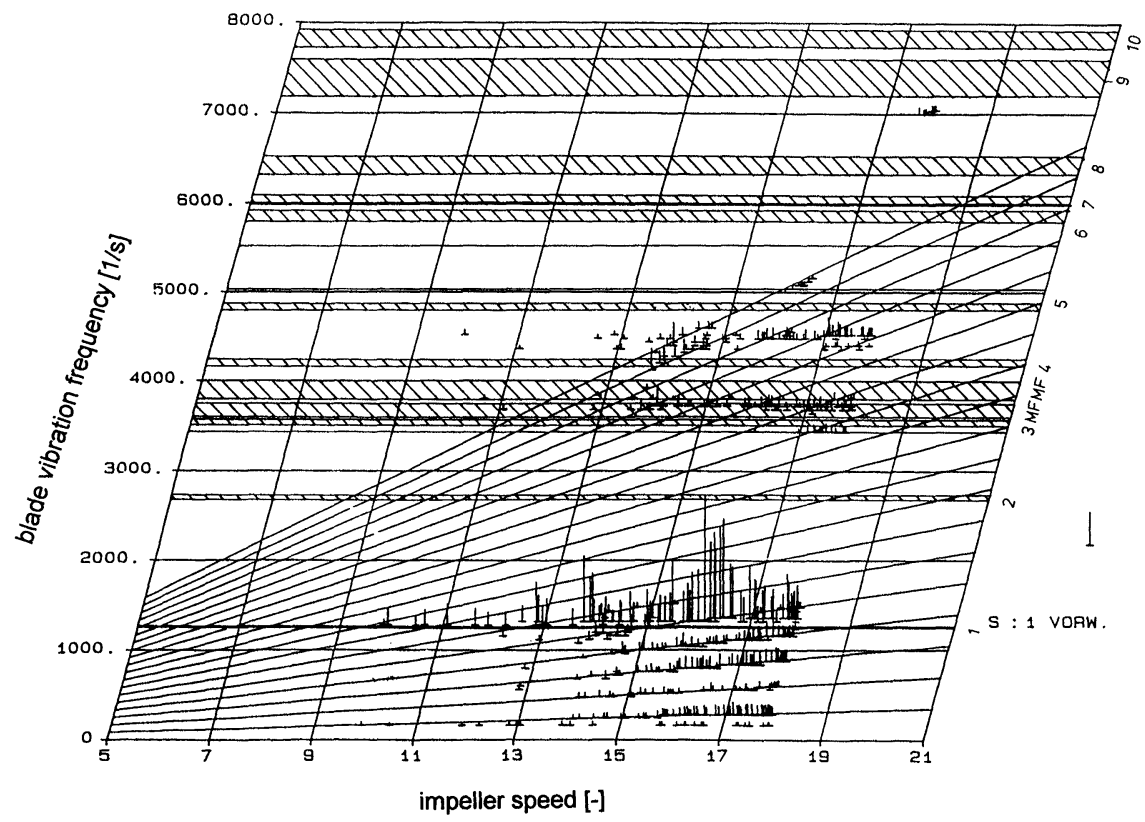

FIGURE 10 Blade vibrations of the 20-bladed $60^{\circ}$-impeller depicted in the Campbell diagram. Results of measurements with a strain gage on the normal blade as well as computed coupled vibration natural frequencies corresponding to the normal blades.

computed coupled vibration natural frequencies corresponding to the normal blade. Owing to the particular point of application of the strain gage the 1 st natural frequency of the blade is very well detected whereas the middle and higher natural frequencies, as already indicated by measurements on the impeller at rest, are only very weakly registered.

The 2nd natural frequency was not detected by the strain gage. Good agreement is obtained, however, between computations and measurements in the case of the 3rd and 4th mode shapes while the mixed form frequencies denoted by MF are also reproduced well by both computations and measurements.

The results of the coupled vibration measurements obtained from a semiconductor strain gage located on the impeller disc in the outlet region between two blades are plotted in the Campbell diagram shown in Fig. 11. Although the 1st and 2nd natural frequencies are only weakly registered by the measurements, good agreement is nevertheless obtained with the FE computations. Also in the range of the mixed forms MF, a good match is obtained between computations and measurements. The 5th natural frequency is also satisfactorily reproduced, whereas in the upper frequency range, it is no longer possible to make any precise prognoses. In overall terms, the amplitudes measured by this strain gage are very small.

\section{Mode Shapes}

The experimental determination of mode shapes was carried out in the same manner as already described in Part I for the $90^{\circ}$-impeller by means of holographic interferometry with the impeller at rest on a small test stand. The results of the natural vibration computations for the complete impeller model were taken as a basis for comparison. A gray scale representation was chosen for depicting the displacements so as to closely resemble the appearance of the interferograms.

A comparison between the measured 1st mode shape and the results of the FE computations for the complete impeller model is shown in Fig. 12. 


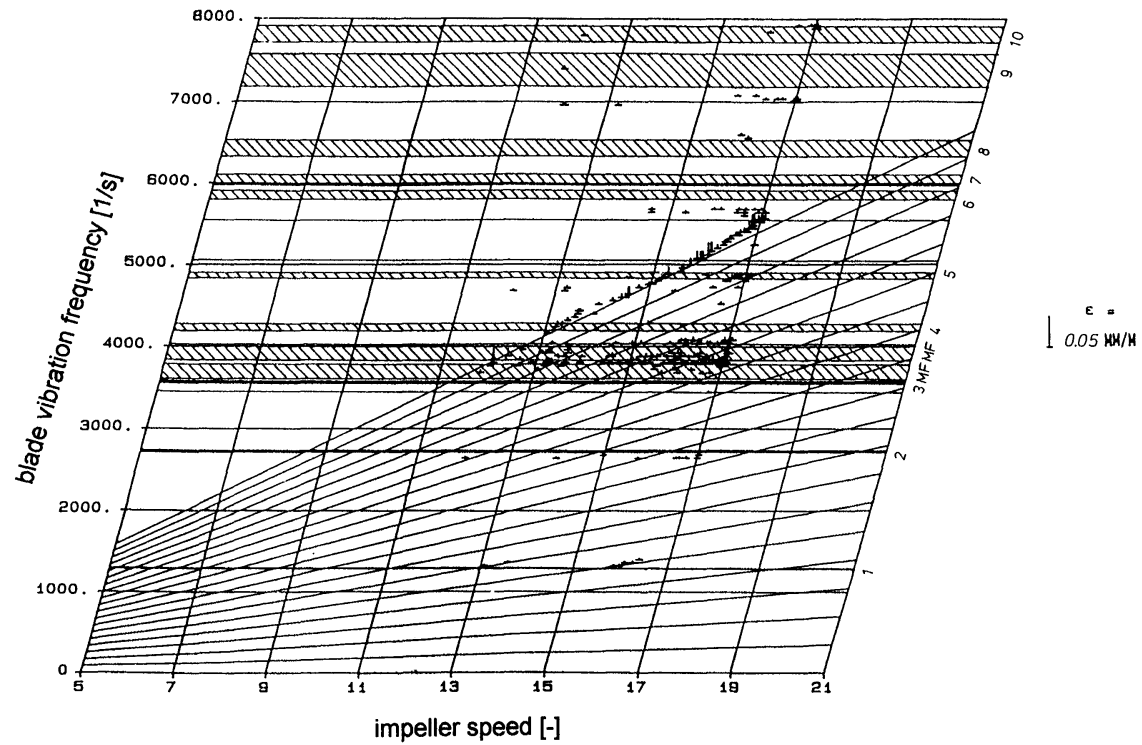

FIGURE 11 Blade vibrations of the 20-bladed $60^{\circ}$-impeller depicted in the Campbell diagram. Results of measurements with a strain gage on the impeller disc as well as computed coupled vibration natural frequencies corresponding to the normal blades.

The interferogram was recorded at a frequency of $1310 \mathrm{~Hz}$ and shows normal blades vibrating in their fundamental mode shape. The varying intensity of the interference fringes indicates that the blades vibrate with different amplitudes. The computed 1st mode shape at $1257 \mathrm{~Hz}$ is depicted in the form of a gray scale representation of the displacement and permits a very good visual comparison to be made with the interferogram. It is clearly evident from the figure that the computations as well as the measured interferogram exhibit different blade vibration amplitudes. These amplitude differences may be explained by coupling effects between the blades. A characteristic feature is the fact that some blades are virtually at rest while neighboring blades vibrate with large amplitudes. These typical coupling effects are well reproduced by both experiments and computations.

A very interesting mode of vibration may also be seen in Fig. 13, which shows an interferogram recorded at $2712 \mathrm{~Hz}$. In this case the normal blades vibrate in their 2 nd mode shape while the splitter blades vibrate in their 1st mode shape. The corresponding natural frequencies of both types of blade lie very close together. The impeller disc also participates in this vibration. The 14 node lines appearing on the disc may be explained by the fact that the corresponding natural frequency of the disc, the mode shape of which contains 14 node lines, lies in very close proximity. Both types of blade as well as the disc vibrate in their natural mode shape in this frequency range. The computed mode shape of the complete impeller model at $2627 \mathrm{~Hz}$, as also shown in Fig. 13, does not indicate any participation of the impeller disc, however. The blade vibrations, on the other hand, are well reproduced.

Of special significance is the interferogram recorded at $3744 \mathrm{~Hz}$, as shown in Fig. 14. The 20 antinodes of the vibrating impeller disc are immediately noticeable in this case. The 20 blades lie on node lines and also exhibit vibrations. Vibration of the splitter blades, which are only partly visible, is also evident. These vibrate in their 2 nd mode shape whereas the normal blades vibrate in their $3 \mathrm{rd}$ mode shape. At this frequency the disc vibrates in its 20th mode shape with 20 node lines. This coupled frequency range was referred to as the mixed form 

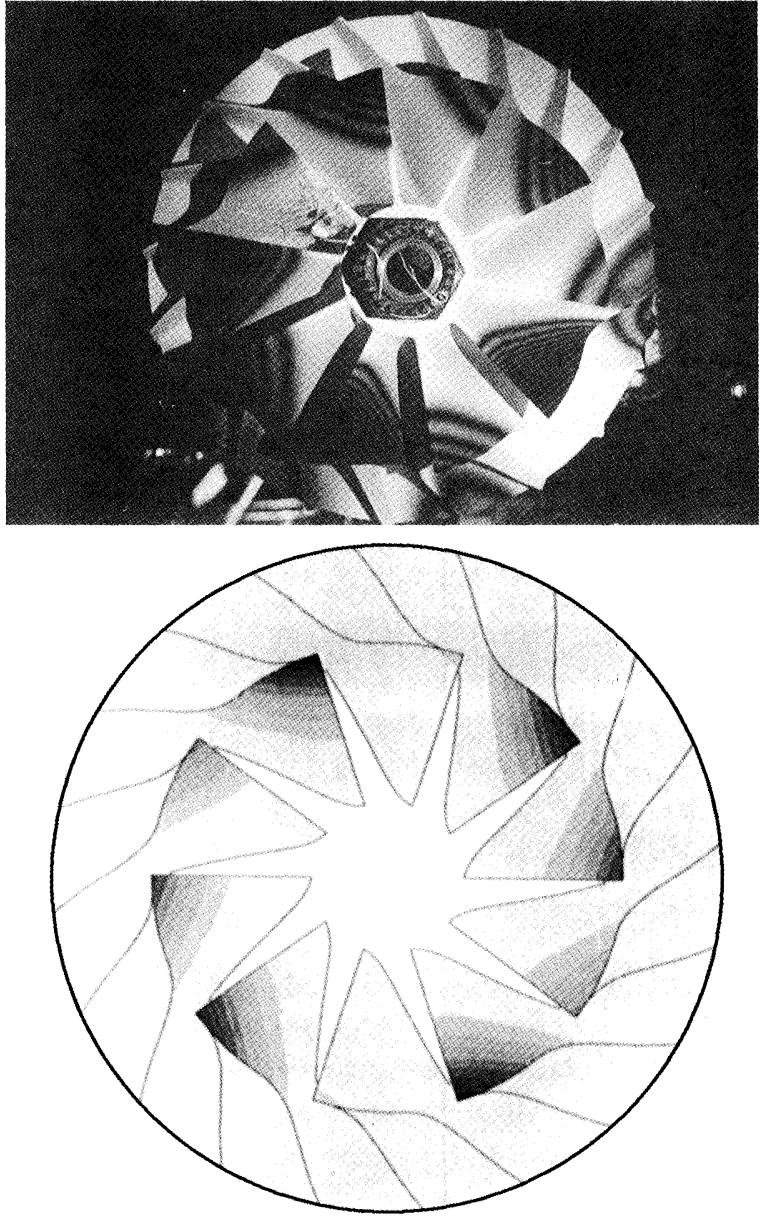

FIGURE 12 Comparison between the holographic interferogram and the computed results for the 7th mode shape of the $60^{\circ}$-impeller with 20 blades.

MF1 in the computations as both types of blade vibrate in their natural mode shapes simultaneously in this case. The corresponding computed mode shape of the complete impeller model at $3532 \mathrm{~Hz}$ exhibits a very similar behavior. In this case, however, the disc vibrates with very small amplitudes. The computed coupled vibration mode shape reproduces the vibration behavior of the impeller very well in this coupled vibration frequency band.

The hologram shown in Fig. 15 was recorded at a frequency of $3955 \mathrm{~Hz}$ and indicates very large amplitudes of the normal blade vibrating in its $3 \mathrm{rd}$ mode shape. The splitter blades in this case vibrate in their 2 nd mode shape. Antinodes are again
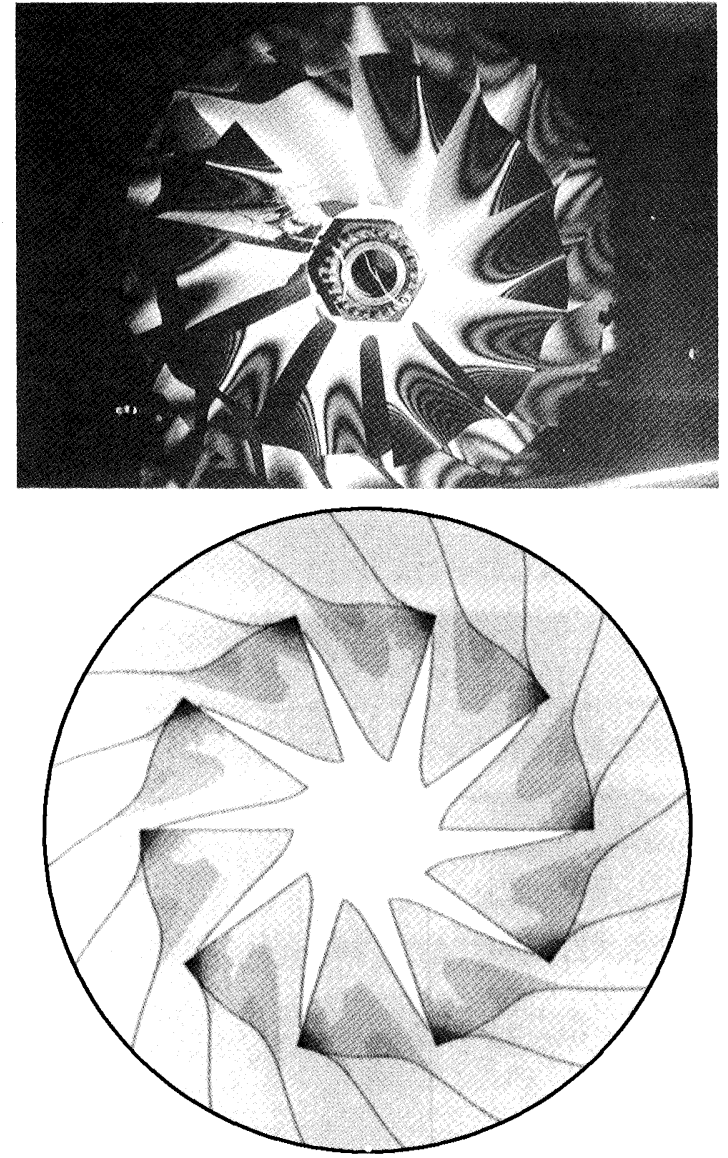

FIGURE 13 Comparison between the holographic interferogram $(2712 \mathrm{~Hz})$ and the computed results $(2627 \mathrm{~Hz})$ for the 21 st mode shape of the $60^{\circ}$-impeller with 20 blades.

evident between the blades on the disc, although they are not as regular as in the case previously considered. As a result of the partly very large amplitudes occurring in this case, the antinodes are found to slightly overlap the interblade region.

This coupled natural frequency band was referred to as the mixed form MF2 in the computations as it is characterized, as in the case of the previously considered mixed frequency band MF1, by resonant vibrations of both types of blade. In close agreement with the hologram, the computed mode shape for the complete impeller model at $3725 \mathrm{~Hz}$ also indicates large vibrations of both the normal and splitter blades. In the case of the 

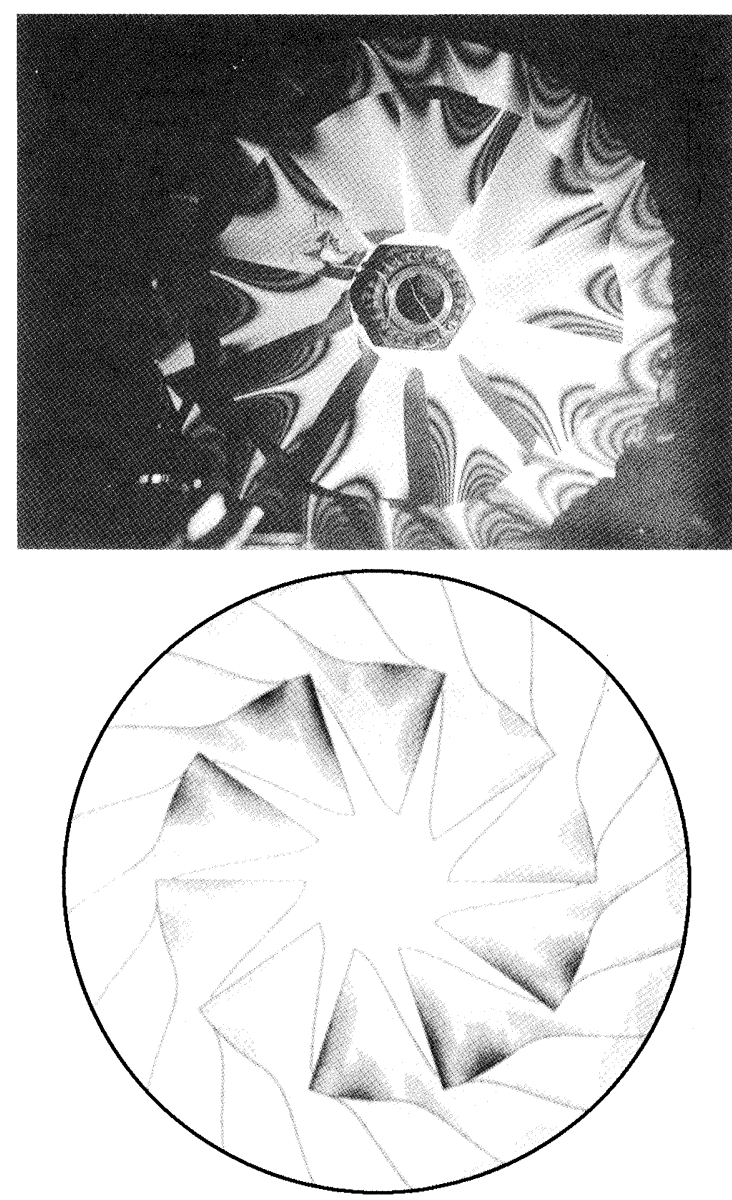

FIGURE 14 Comparison between the holographic interferogram $(3744 \mathrm{~Hz})$ and the computed results $(3532 \mathrm{~Hz})$ for the 31 st mode shape of the $60^{\circ}$-impeller with 20 blades.

disc, however, antinodes are only present in every second channel.

The interferogram presented in Fig. 16 shows the coupled vibration behavior of the impeller at $4401 \mathrm{~Hz}$. This clearly indicates large vibrations of the normal blades in their 4th mode shape. The splitter blades, which are partly hidden by the normal blades, also exhibit large displacements over their visible length. It may be deduced from the latter that this type of blade vibrates in its 2 nd mode shape, the frequency of which lies in very close proximity. The disc also exhibits pronounced vibrations which differ with location and whose node lines no longer coincide with the blade paths
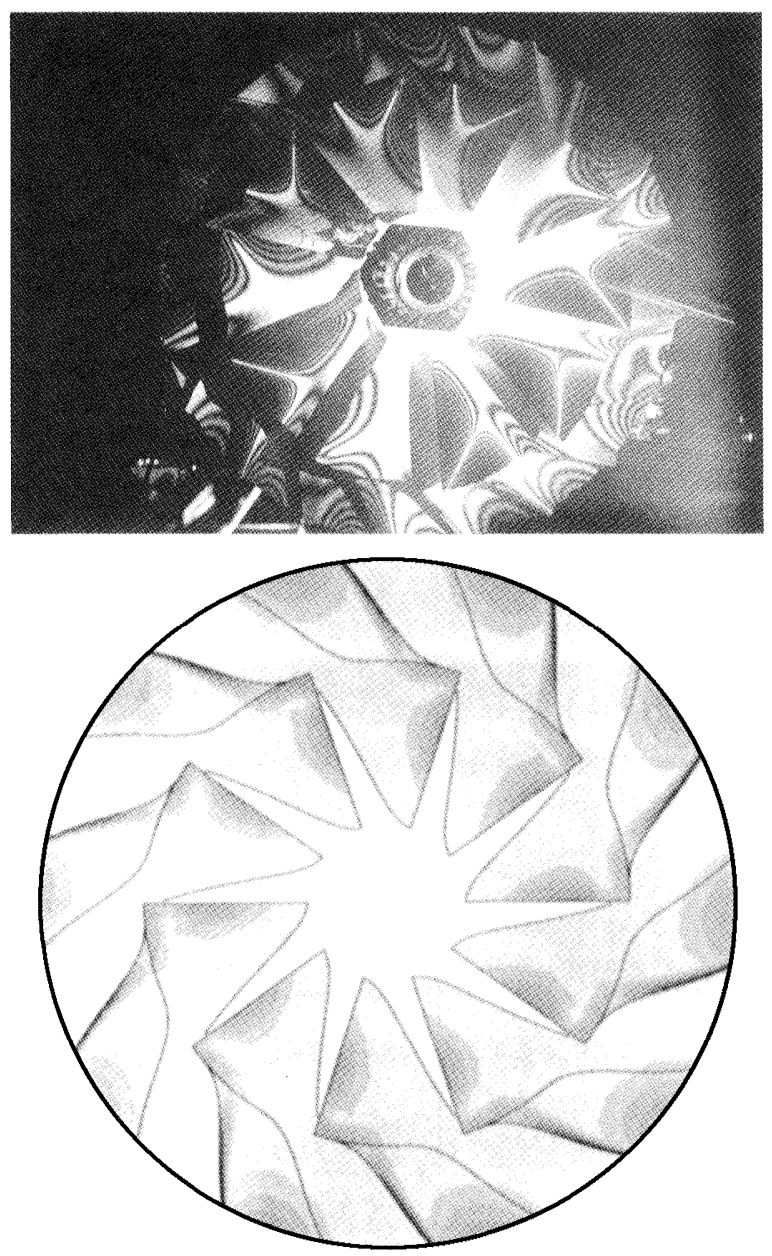

FIGURE 15 Comparison between the holographic interferogram $(3955 \mathrm{~Hz})$ and the computed results $(3725 \mathrm{~Hz})$ for the 50 th mode shape of the $60^{\circ}$-impeller with 20 blades.

in all cases. The computed mode shape for the complete impeller model at $4180 \mathrm{~Hz}$ also reveals high amplitudes at the tip of the normal blade at the outlet and a pronounced node line running from the outer profile over a quarter circle around the blade tip to the leading edge. A further antinode is evident along the outer profile as well as on the leading edge. A further node line runs behind these antinodes obliquely forwards towards the blade root at the inlet while a further antinode is present along the outer profile about half way along the blade length. As indicated by the interferogram, the impeller disc also participates in the coupled 

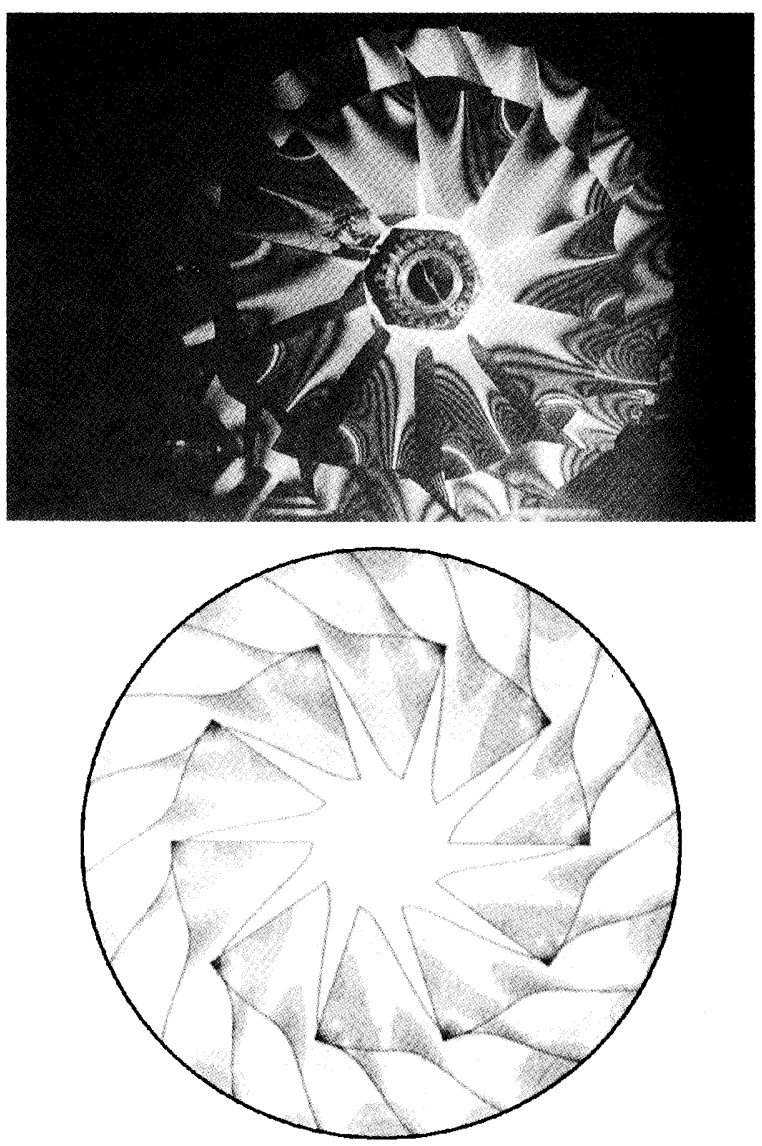

FIGURE 16 Comparison between the holographic interferogram $(4401 \mathrm{~Hz})$ and the computed results $(4180 \mathrm{~Hz})$ for the 61 st mode shape of the $60^{\circ}$-impeller with 20 blades.

vibration over its entire circumference. The computed results also yield antinodes over the entire circumference of the disc, which alternately vary in amplitude from large to small.

\section{CONCLUSIONS}

The coupled vibration computations for the $60^{\circ}$ impeller also confirm that the FE method is a powerful tool for computing natural frequencies as well as mode shapes. Comparisons between experimentally determined vibration behavior and the results obtained by the FE method were found to be in good agreement in most cases. Deviations on the experimental side could be explained by unfavorable positioning of the strain gages, whereas on the computing side, inaccuracies were largely due to the coarse discretization of the models, particularly in the higher frequency range. This situation may be improved by a finer discretization of the models. Even with the model used in the present investigation, however, it was still possible to obtain good prognoses of coupled vibration behavior. Also in the case of the $60^{\circ}$-impeller, the experiments and computations clearly demonstrated the need to properly account for the flexible part of the impeller disc when determining coupled vibration mode shapes. Strictly speaking, the isolated computation of rigidly fixed individual structures can only exactly reproduce the fundamental mode of vibration. This approach, however, is unable to simulate the coupling effects between the disc and the blades, as characterized by frequency shifts and frequency band spreading.

A noticeable feature compared with the results for the $90^{\circ}$-impeller, as was clearly evident in the interferograms, is the fact that the impeller disc plays a far more dominant role in the coupled vibration behavior.

Even above the 2nd mode shape of the normal blade, pronounced impeller blade vibrations occurred which led to increased mutual interactions between the normal and splitter blades. It was thus found that both types of blade participate in the majority of middle and higher coupled natural vibrations. This behavior was found to be mostly dominated by one blade type while the other blade type was caused to vibrate through forced excitations. This fact may be explained by the considerably stronger attachment of the $60^{\circ}$-blades to the impeller disc. Besides the mutual interaction via moments, as is predominant in the case of the $90^{\circ}$-impeller, forces between the blades and the disc are also transferred via translatory movements in the case of the $60^{\circ}$ impeller, i.e. via displacements in the axial direction. This effect may be explained by the backswept nature of the structure and is absent in the case of radial-ending blades, which run purely radially at the point of connection with the impeller disc. 
It may be concluded from the foregoing that the risk of material damage due to coupled vibrations is greater for a centrifugal impeller with backswept blades than for an impeller with radial-ending blades.

\section{Acknowledgements}

The authors gratefully acknowledge the financial support of the German Research Association (DFG) throughout this research project and also express their gratitude to the Lower Saxony Regional Computing Center (RRZN) for providing the computing facilities and the finite element software. They would also like to thank Dipl.-Ing. Bubbel and Mr. Wichmann for their cooperation as well as Mr. Tanneberg for running the tests and Mr. Wichmann for conducting the measurements. The authors are also indebted to Dr. Ian Westwood (Burgwedel, Germany) for his meticulous translation of this paper.

\section{NOMENCLATURE}

\section{Symbols}

$A$ amplitude

$x \quad$ coordinate
$c \quad$ spring constant
$M$ mass
$t$ time
$\omega \quad$ natural frequency

\section{Subscripts}

1 mass 1

2 mass 2

$\mathrm{k} \quad$ coupled

\section{References}

Haupt, U. (1984) Untersuchung des Schaufelschwingungsverhaltens hochbelasteter Radialverdichterlaufräder, Dissertation, Universität Hannover.

Jin, D. (1990) Untersuchung von Schaufelschwingungen und ihrer Erregungsursachen an Radialverdichtern, Dissertation, Universität Hannover.

Rautenberg, M., Engeda, A. and Wittekind, W. (1989a) Mathematical formulation of blade surfaces in turbomachinery, Part I: Theoretical surface formulations, ASME- Paper No. 89-GT-160, 34th ASME Int. Gas Turbine and Aeroengine Congress and Exposition, Toronto, Ontario, Canada.

Rautenberg, M., Engeda, A. and Wittekind, W. (1989b) Mathematical formulation of blade surfaces in turbomachinery, Part II. Practical examples of determined surfaces, ASME-Paper No. 89-GT-161, 34th ASME Int. Gas Turbine and Aeroengine Congress and Exposition, Toronto, Ontario, Canada. 


\section{ait \\ ENERGY MATERIALS}

M A N E Y publishing

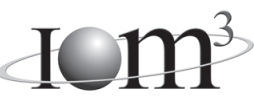

\section{Materials Science \& Engineering for Energy Systems}

Maney Publishing on behalf of the Institute of Materials, Minerals and Mining

The Institute of Materials, Minerals \& Mining

Economic and environmental factors are creating ever greater pressures for the efficient generation, transmission and use of energy. Materials developments are crucial to progress in all these areas: to innovation in design; to extending lifetime and maintenance intervals; and to successful operation in more demanding environments. Drawing together the broad community with interests in these areas, Energy Materials addresses materials needs in future energy generation, transmission, utilisation, conservation and storage. The journal covers thermal generation and gas turbines; renewable power (wind, wave, tidal, hydro, solar and geothermal); fuel cells (low and high temperature); materials issues relevant to biomass and biotechnology; nuclear power generation (fission and fusion); hydrogen generation and storage in the context of the 'hydrogen economy'; and the transmission and storage of the energy produced.

As well as publishing high-quality peer-reviewed research, Energy Materials promotes discussion of issues common to all sectors, through commissioned reviews and commentaries. The journal includes coverage of energy economics and policy, and broader social issues, since the political and legislative context influence research and investment decisions.

\section{CALL FOR PAPERS}

Contributions to the journal should be submitted online at http://ema.edmgr.com

To view the Notes for Contributors please visit: www.maney.co.uk/journals/notes/ema

Upon publication in 2006, this journal will be available via the Ingenta Connect journals service. To view free sample content online visit: www.ingentaconnect.com/content/maney

For further information please contact:

Maney Publishing UK

Tel: +44 (0)113 2497481 Fax: +44 (0)1132486983 Email: subscriptions@maney.co.uk

or

Maney Publishing North America

Tel (toll free): 8662975154 Fax: 6173546875 Email: maney@maneyusa.com

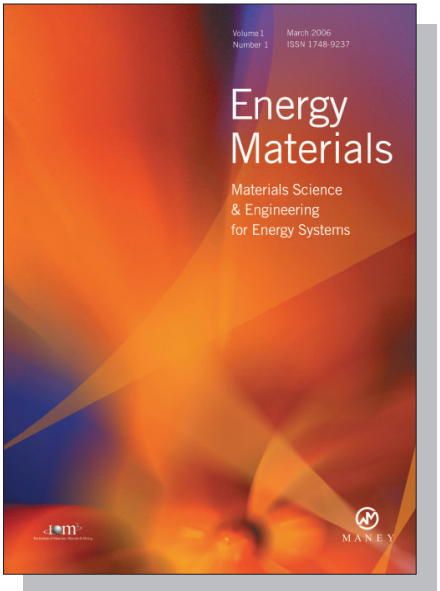

EDITORS

Dr Fujio Abe

NIMS, Japan

Dr John Hald, IPL-MPT, Technical University of Denmark, Denmark

Dr R Viswanathan, EPRI, USA

\section{SUBSCRIPTION INFORMATION}

Volume 1 (2006), 4 issues per year

Print ISSN: 1748-9237 Online ISSN: 1748-9245

Individual rate: $£ 76.00 / U S \$ 141.00$

Institutional rate: $£ 235.00 /$ US $\$ 435.00$

Online-only institutional rate: $£ 199.00 / U S \$ 367.00$

For special $\mathrm{IOM}^{3}$ member rates please email

subscriptions@maney.co.uk

\section{For further information or to subscribe online please visit www.maney.co.uk}



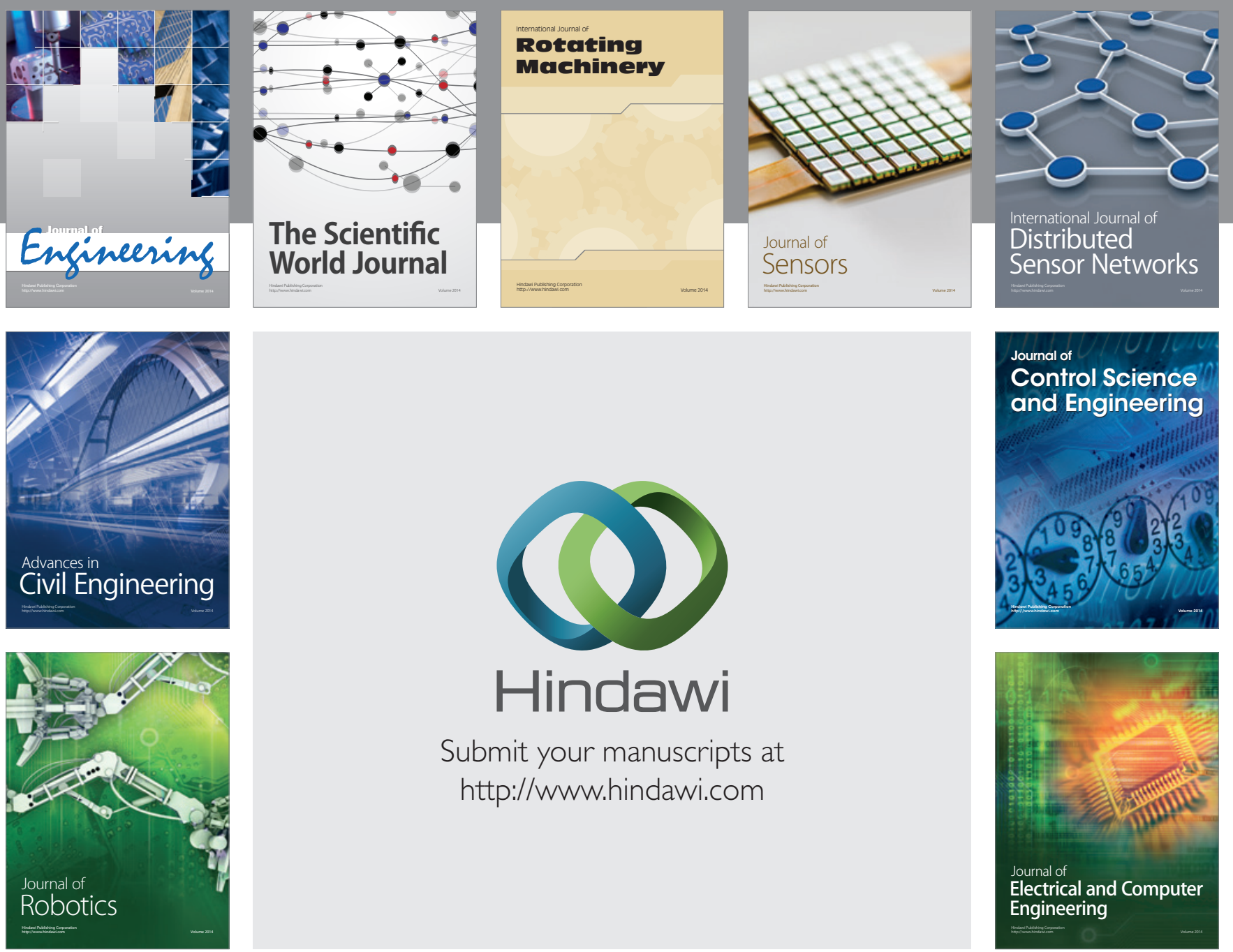

Submit your manuscripts at

http://www.hindawi.com
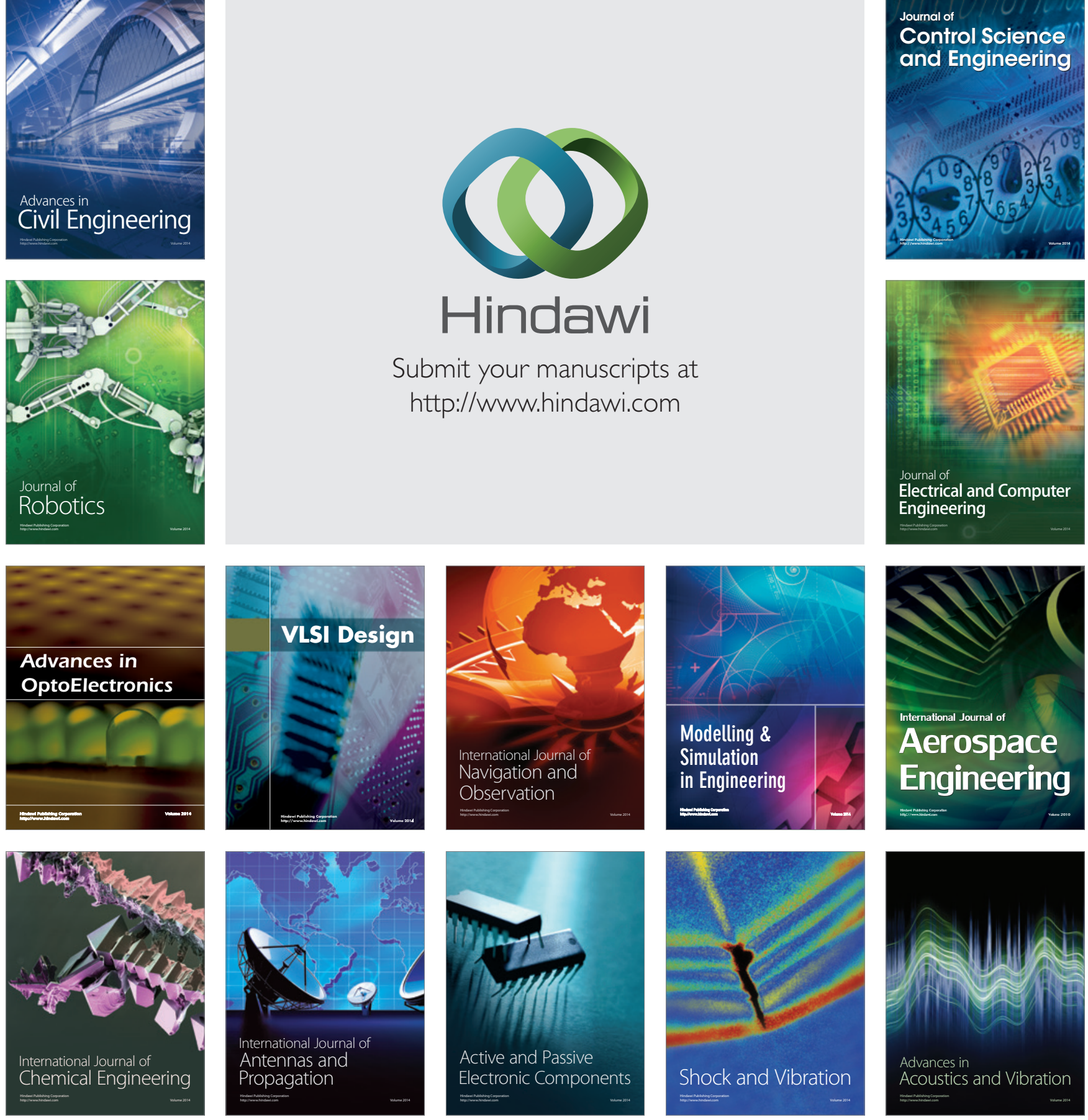